\title{
Trends in sexual behaviour among London homosexual men 1998-2003: implications for HIV prevention and sexual health promotion
}

\author{
J Elford, G Bolding, M Davis, L Sherr, G Hart
}

Sex Transm Infect 2004;80:451-454. doi: 10.1136/sti.2004.010785

\begin{abstract}
Objectives: To examine changes in sexual behaviour among London homosexual men between 1998 and 2003 by type and HIV status of partner.

Methods: Homosexual men $(n=4264)$ using London gyms were surveyed annually between 1998 and 2003 (range 498-834 per year). Information was collected on HIV status, unprotected anal intercourse (UAl) in the previous 3 months, and type of partner for UAl. High risk sexual behaviour was defined as UAI with a partner of unknown or discordant HIV status.

Results: Between 1998 and 2003, the percentage of men reporting high risk sexual behaviour with a casual partner increased from $6.7 \%$ to $16.1 \%$ (adjusted odds ratio (AOR) 1.36 per year, $95 \%$ confidence interval (Cl) 1.26 to $1.46, p$ $<0.001)$. There was no significant change in the percentage of men reporting high risk sexual behaviour with a main partner alone $(7.8 \%, 5.6 \%, p=0.7)$. These patterns were seen for HIV positive, negative and never tested men alike regardless of age. The percentage of HIV positive men reporting UAl with a casual partner who was also HIV positive increased from $6.8 \%$ to $10.3 \%$ (AOR $1.27,95 \% \mathrm{Cl}$ 1.01 to $1.58, \mathrm{p}<0.05)$.

Conclusion: The increase in high risk sexual behaviour among London homosexual men between 1998 and 2003 was seen only with casual and not with main partners. STI/ HIV prevention campaigns among London homosexual men should target high risk practices with casual partners since these appear to account entirely for the recent increase in high risk behaviour.
\end{abstract}

$\mathrm{T}$ he past decade has seen substantial increases in high risk sexual behaviour in the British population. ${ }^{1}$ Diagnoses of chlamydia, gonorrhoea, and syphilis have more than doubled in the last 5 years. ${ }^{2}$ Certain "core groups" have been at particular risk of sexually transmitted infections (STI). For example, there have been several large outbreaks of syphilis in England over the last few years, notably in Manchester, Brighton, and London, which have predominantly involved homosexual men, many of whom were also infected with HIV. ${ }^{2}$ Homosexual men are also the group at highest risk of acquiring HIV in the United Kingdom despite the growing number of new HIV diagnoses by heterosexual transmission. ${ }^{3}$ STI and HIV prevention remain, therefore, a priority for homosexual men in the United Kingdom, including men with HIV infection. Formulating effective interventions, however, requires a sound evidence base concerning sexual practices. For this reason, we examined changes in sexual behaviour among London homosexual men between 1998 and 2003 by HIV status of respondent and type and HIV status of their sexual partners.

\section{METHODS \\ Data collection}

Homosexual/bisexual men who use London gyms have been surveyed annually since 1998 as part of a behavioural surveillance programme. ${ }^{4}$ Each year, between January and March, men are asked to complete a confidential self administered questionnaire providing information on social and demographic characteristics, HIV status, self reported sexual behaviour and, from 1999, participation in previous gym surveys. Men are asked whether they had unprotected anal intercourse (UAI) in the previous 3 months and, if so, the type (main or casual) and HIV status of their UAI partner(s). UAI is classified as either concordant (only with a partner of the same HIV status) or non-concordant (with a partner of unknown or discordant HIV status). In this analysis, concordant and non-concordant UAI are mutually exclusive categories. ${ }^{4}$ Non-concordant UAI clearly presents a risk for HIV transmission. Men who reported UAI only with a main partner were analysed separately from those who reported UAI with casual partners.

\section{Data analysis}

To analyse time trends in sexual risk behaviour we first examined, in a logistic model, the univariate association between year of survey and UAI for all men and then by HIV status of respondent. In this model the independent variable was year of survey (entered as a dummy variable $1998=0$, $1999=1,2000=2$, etc) while the dependent variable was UAI. Separate analyses were conducted for concordant UAI and non-concordant UAI broken down by type of partnermain only or casual.

When a significant univariate association was found between year of survey and UAI, the multivariate association was examined by simultaneously entering independent, dependent and confounding variables into the logistic model. The adjusted odds ratio derived from this model measured the increase from one year to the next in the likelihood of reporting UAI after controlling for confounding factors. Confounding factors were those variables which were significantly associated with both UAI and year of survey. Overall, there was little variation in the background characteristics of the study group between 1998 and 2003; in all years the majority of men were white (1998 to 2003 combined, $90 \%$ ), employed (85\%), and university educated $(78 \%)$. To identify potential confounding factors, the relation between year of survey and variables known to be associated with high risk sexual behaviour in this sample was examined.

Abbreviations: HAART, highly active antiretroviral therapy; STI, sexually transmitted infections; UAl, unprotected anal intercourse 
Table 1 Number of men surveyed between 1998 and 2003 by HIV status

\begin{tabular}{|c|c|c|c|c|c|c|c|c|c|c|c|c|c|c|c|c|}
\hline & \multicolumn{2}{|c|}{1998} & \multicolumn{2}{|c|}{1999} & \multicolumn{2}{|c|}{2000} & \multicolumn{2}{|c|}{2001} & \multicolumn{2}{|c|}{2002} & \multicolumn{2}{|c|}{2003} & \multicolumn{2}{|l|}{ Total } & \multicolumn{2}{|c|}{$\begin{array}{l}\text { Independent } \\
\text { samples }\end{array}$} \\
\hline & No & $\%$ & No & $\%$ & No & $\%$ & No & $\%$ & No & $\%$ & No & $\%$ & No & $\%$ & No & $\%$ \\
\hline HIV positive men & 118 & 14.1 & 101 & 16.0 & 120 & 16.2 & 116 & 15.8 & 121 & 14.6 & 78 & 15.7 & 654 & 15.3 & 387 & 14.6 \\
\hline HIV negative men & 483 & 57.9 & 396 & 62.9 & 459 & 62.1 & 438 & 59.6 & 542 & 65.5 & 334 & 67.1 & 2652 & 62.2 & 1649 & 62.4 \\
\hline Never tested men & 233 & 27.9 & 133 & 21.1 & 160 & 21.7 & 181 & 24.6 & 165 & 19.9 & 86 & 17.3 & 958 & 22.5 & 60 & 23.0 \\
\hline All men & 834 & 100.0 & 630 & 100.0 & 739 & 100.0 & 735 & 100.0 & 828 & 100.0 & 498 & 100.0 & 4264 & 100.0 & 2644 & 100.0 \\
\hline
\end{tabular}

Variables associated with high risk sexual behaviour in this sample are age, being in a relationship, steroid use, ${ }^{56}$ and HIV treatments optimism. ${ }^{4}$ Where these variables fluctuated from year to year, the multivariate model was adjusted accordingly. The multivariate model excluded men who had completed a questionnaire in a previous year to ensure independence of samples.

\section{RESULTS}

Complete information on HIV status, sexual risk behaviour, and potential confounders was provided by 4264 men over the study period: HIV positive $654(15.3 \%)$, HIV negative $2652(62.2 \%)$, never tested 958 (22.5\%) (range 498-834 per year, estimated response rate $50-60 \%,{ }^{4}$ table 1). After excluding men who had completed a previous questionnaire 2644 respondents remained for the independent samples analysis. Between 1998 and 2003, for the study group as a whole, there was a significant increase in the percentage of men reporting both non-concordant UAI ( $14.5 \%$ to $21.7 \%$, $\mathrm{p}<0.001)$ and concordant UAI $(9.8 \%$ to $14.7 \%$, p < $<0.001)$ (table 2). The majority of men reporting non-concordant UAI said they did not know the HIV status of their UAI partner but had assumed it was the same as theirs. Only a minority of men said they knew that their UAI partner was discordant. For example, in 2002181 men $(21.9 \%)$ reported nonconcordant UAI; 167 men (20.2\% of the sample) reported UAI with a man whose HIV status they didn't know while 14 men $(1.7 \%)$ said their UAI partner was discordant.

\section{Non-concordant UAI}

The increase in non-concordant UAI was seen only with casual partners $(6.7 \%$ to $16.1 \%, p<0.001)$ (table 2 , fig 1 ). There was no significant trend over time for non-concordant UAI with a main partner alone $(7.8 \%, 5.6 \% \mathrm{p}=0.7)$. These patterns were seen for HIV positive, HIV negative and nevertested men alike. In multivariate analysis, after controlling for confounding factors (HIV treatments optimism, steroid use and relationship status), the increase in non-concordant UAI with a casual partner remained statistically significant for all men (adjusted odds ratio (AOR) 1.36 per year, 95\% confidence interval (CI) 1.26 to $1.46, \mathrm{p}<0.001$ ) and for HIV positive, HIV negative, and never tested men when analysed separately $(\mathrm{p} \leqslant 0.01)$ (table 2$)$

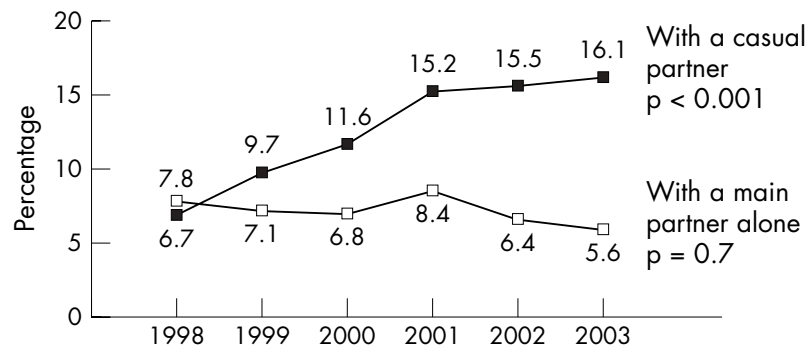

Figure 1 Percentage of men reporting non-concordant unprotected anal intercourse (UAl) between 1998 and 2003.

Table 2 Number (\%) of men reporting unprotected anal intercourse (UAI) between 1998 and 2003

\begin{tabular}{|c|c|c|c|c|c|c|c|c|c|c|c|c|c|c|c|}
\hline & \multicolumn{2}{|c|}{1998} & \multicolumn{2}{|c|}{1999} & \multicolumn{2}{|c|}{2000} & \multicolumn{2}{|c|}{2001} & \multicolumn{2}{|c|}{2002} & \multicolumn{2}{|c|}{2003} & \multirow{2}{*}{$\begin{array}{l}\text { Odds } \\
\text { ratio }\end{array}$} & \multirow[b]{2}{*}{$95 \% \mathrm{Cl}$} & \multirow[b]{2}{*}{ p Value } \\
\hline & No & $\%$ & No & $\%$ & No & $\%$ & No & $\%$ & No & $\%$ & No & $\%$ & & & \\
\hline Non-concordant UAI & & & & & & & & & & & & & & & \\
\hline $\begin{array}{l}\text { All non-concordant UAI } \\
\text { With a casual partner }\end{array}$ & 121 & 14.5 & 106 & 16.8 & 136 & 18.4 & 174 & 23.6 & 181 & 21.9 & 108 & 21.7 & 1.24 & $1.16-1.31$ & $<0.001$ \\
\hline HIV positive men & 18 & 15.3 & 15 & 14.9 & 23 & 19.2 & 45 & 38.8 & 50 & 41.3 & 29 & 37.2 & 1.46 & $1.26-1.69$ & $<0.001$ \\
\hline HIV negative men & 33 & 6.8 & 40 & 10.1 & 49 & 10.7 & 53 & 12.1 & 65 & 12.0 & 47 & 14.1 & 1.32 & $1.20-1.46$ & $<0.001$ \\
\hline Never tested men & 5 & 2.1 & 6 & 4.5 & 14 & 8.8 & 14 & 7.7 & 13 & 7.9 & 4 & 4.7 & 1.28 & $1.06-1.54$ & 0.01 \\
\hline $\begin{array}{l}\text { All men } \\
\text { With main partner alone }\end{array}$ & 56 & 6.7 & 61 & 9.7 & 86 & 11.6 & 112 & 15.2 & 128 & 15.5 & 80 & 16.1 & 1.36 & $1.26-1.46$ & $<0.001$ \\
\hline HIV positive men & 5 & 4.2 & 5 & 5.0 & 2 & 1.7 & 5 & 4.3 & 1 & 0.8 & 2 & 2.6 & - & - & 0.7 \\
\hline HIV negative men & 29 & 6.0 & 26 & 6.6 & 25 & 5.4 & 34 & 7.8 & 32 & 5.9 & 18 & 5.4 & - & - & 0.7 \\
\hline Never tested men & 31 & 13.3 & 14 & 10.5 & 23 & 14.4 & 23 & 12.7 & 20 & 12.1 & 8 & 9.3 & - & - & 0.7 \\
\hline $\begin{array}{l}\text { All men } \\
\text { Concordant UAl }\end{array}$ & 65 & 7.8 & 45 & 7.1 & 50 & 6.8 & 62 & 8.4 & 53 & 6.4 & 28 & 5.6 & - & - & 0.7 \\
\hline $\begin{array}{l}\text { All concordant UAI } \\
\text { With a casual partner }\end{array}$ & 82 & 9.8 & 76 & 12.1 & 113 & 15.3 & 81 & 11.0 & 126 & 15.2 & 73 & 14.7 & 1.16 & $1.08-1.25$ & $<0.001$ \\
\hline HIV positive men & 8 & 6.8 & 4 & 4.0 & 17 & 14.2 & 13 & 11.2 & 11 & 9.1 & 8 & 10.3 & 1.27 & $1.01-1.58$ & 0.04 \\
\hline $\begin{array}{l}\text { HIV negative men } \\
\text { With main partner alone }\end{array}$ & 8 & 1.7 & 5 & 1.3 & 9 & 2.0 & 4 & 0.9 & 15 & 2.8 & 7 & 2.1 & - & - & 0.2 \\
\hline HIV positive men & 6 & 5.1 & 6 & 6.0 & 7 & 5.8 & 6 & 5.2 & 7 & 5.8 & 5 & 6.4 & - & - & 0.2 \\
\hline HIV negative men & 60 & 12.4 & 61 & 15.4 & 80 & 17.4 & 58 & 13.2 & 93 & 17.2 & 53 & 15.9 & 1.11 & $1.02-1.21$ & 0.02 \\
\hline
\end{tabular}

Odds ratios from multivariate model.

The percentages in table 2 were derived by dividing the number of men reporting UAl by the number of men surveyed (table 1)- for example, for HIV positive men reporting non-concordant UAl with a casual partner in 1998 the percentage is $18 / 118=15.3 \%$. 
In all years HIV positive men were more likely to report non-concordant UAI with a casual partner than with a main partner alone $(p<0.001)$. Never tested men, on the other hand, were more likely to report non-concordant UAI with a main partner alone rather than with a casual partner $(p<0.001)$. For HIV negative men the pattern varied over time. In 1998 HIV negative men were just as likely to report non-concordant UAI with a main partner as with a casual partner $(6.0 \% v 6.8 \%, p=0.9)$. By 2003 , however, they were more likely to report non-concordant UAI with a casual partner $(14.1 \% \vee 5.4 \%$, test for a trend, $\mathrm{p}<0.05)$.

\section{Concordant UAI}

The percentage of men reporting concordant UAI with a casual partner increased significantly between 1998 and 2003 for HIV positive men $(6.8 \%$ to $10.3 \%, \mathrm{p}<0.05)$ but not for HIV negative men $(1.7 \%, 2.1 \%, \mathrm{p}=0.2)$ (table 2 ).

The percentage of men reporting concordant UAI with a main partner alone increased significantly between 1998 and 2003 for HIV negative men ( $12.4 \%$ to $15.9 \%$, p < 0.05 ) but not for HIV positive men $(5.1 \%, 6.4 \%, p=0.2$ ) (table 2 ). However, preliminary inspection of data for $2004^{8}$ indicates that the percentage of HIV negative men reporting concordant UAI with a main partner alone dropped to $12.8 \%$. As a consequence there was no significant trend over time between 1998 and 2004.

\section{DISCUSSION}

Our findings have important implications for STI and HIV prevention. The increase in high risk sexual behaviour among London homosexual men between 1998 and 2003 was seen only with casual partners and not with main partners. STI/ HIV interventions among these men should therefore target high risk practices with casual partners since these appear to account entirely for the recent increase in high risk sexual behaviour. The factors underlying this increase are not fully understood. ${ }^{9}$ None the less, establishing that it has occurred only with casual partners will assist health promotion agencies in formulating their prevention strategies.

Most of the men who reported non-concordant UAI in our study said they did not know the HIV status of their sexual partner but had assumed it was the same as theirs; only a minority reported UAI with a partner they knew to be discordant. This suggests that homosexual men are unlikely to engage in UAI when they know for a fact that HIV transmission can occur. They may do so, however, in situations where they believe that transmission is unlikely to occur, by assuming they share the same HIV status as their casual partner.

The increase in non-concordant UAI reported here not only reflects that seen among homosexual men surveyed in bars, clubs, and genitourinary medicine clinics in London ${ }^{10}{ }^{11}$ but also mirrors a substantial rise in the number of new cases of gonorrhoea and syphilis diagnosed among London homosexual men since $1998 .^{2}$

Between 1998 and 2003, an increasing percentage of men diagnosed with HIV reported UAI with casual partners who were also HIV positive. While HIV positive men may mutually disclose their HIV status in a casual encounter they can not, by definition, disclose an undiagnosed STI. Consequently, concordant UAI among HIV positive men presents a risk for STI transmission as well as cross infection with other, potentially drug resistant strains of HIV. ${ }^{12}$ The increase in concordant UAI with casual partners seen here among HIV positive men matches a corresponding increase in STIs, also among HIV positive men, reported by the $\mathrm{HPA}^{2}$ and merits targeted testing, treatment and preventive interventions.

How can we explain the increase in high risk sexual behaviour among homosexual men in London since 1998? An

\section{Key messages}

- There has been a significant increase in the proportion of London homosexual men reporting high risk sexual behaviour since 1998

- This increase was seen only with casual and not with main partners

- STI/HIV prevention among homosexual men should target high risk sexual practices with casual partners

increase that has also been reported in major cities across Europe, Australia, Canada, and the United States. ${ }^{4}$ While the increase has coincided with the introduction of highly active antiretroviral therapy (HAART), it is unlikely that optimism in the light of new treatments can explain the increase at a population level. ${ }^{4}{ }^{13}{ }^{14}$ Other contributory factors could be the increased opportunity in recent years for meeting sexual partners in saunas, backrooms, or through the internet. It is also possible that homosexual men have become habituated to the risk of HIV now that more than two decades have passed since AIDS was first reported. Increasing demand for HIV treatment and care in the era of HAART may also have placed pressure on HIV prevention services..$^{15}$ Clearly, priority should be given to research which helps us to better understand the factors that underlie high risk sexual behaviour among homosexual and bisexual men living in London. Such research should focus on different risk profiles such as HIV status, ethnicity, and socioeconomic status. ${ }^{16}$ This in turn will provide an evidence base for formulating and evaluating HIV/STI preventive interventions.

\section{ACKNOWLEDGEMENTS}

The authors would like to thank the managers and members of the gyms for their support and participation in the project; Edith Stokes and the staff at Mount Pleasant, Surrey, where this manuscript was first drafted. The research was funded by Camden and Islington Health Authority and Primary Care Trusts with additional support from the UK Medical Research Council (grant number GO100159).

\section{CONTRIBUTORS}

All authors contributed to the design of the study; GB was responsible for project administration, data collection and analysis with input from JE; JE wrote the first draft and coordinated subsequent revisions; all authors read the manuscript, suggested revisions and approved the final version; JE is the guarantor

\section{Authors' affiliations \\ J Elford, G Bolding, M Davis, City University London, Institute of Health Sciences, St Bartholomew School of Nursing and Midwifery, London, UK L Sherr, Royal Free and University College Medical School, London, UK G Hart, MRC Social and Public Health Sciences Research Unit, Glasgow, UK}

Correspondence to: Jonathan Elford, City University, Institute of Health Sciences, St Bartholomew School of Nursing and Midwifery, 24 Chiswell Street, London ECIY 4TY, UK; i.elford@city.ac.uk

Accepted for publication 17 July 2004

\section{REFERENCES}

1 Johnson A, Mercer C, Erens B, et al. Sexual behaviour in Britain: partnerships, practices and HIV risk behaviours. Lancet 2001:358:1835-42.

2 PHLS, DHSS\&PS, Scottish ISD(D)5 Collaborative Group. Sexually transmitted infections in the UK: new episodes seen at genitourinary medicine clinics, 1991 to 2001. London: Public Health Labratory Service, 2002.

3 PHLS. AIDS/HIV quarterly surveillance tables. Cumulative UK data to end September 2003. 60 03/3, 2003.

4 Elford J, Bolding G, Sherr L. High risk sexual behaviour increases among London gay men: what is the role of HIV optimism? AIDS 2002;16:1537-44. 
5 Bolding G, Sherr L, Maguire M, et al. HIV risk behaviours among gay men who use anabolic steroids. Addiction 1999;94:1829-35.

6 Bolding G, Sherr L, Elford J. Use of anabolic steroids and associated health risks among gay men attending London gyms. Addiction 2002:97:195-203.

7 Elford J, Bolding G, Maguire M, et al. Combination therapies for HIV and sexual risk behavior among gay men. J Acquir Immune Defic Syndr 2000;23:266-71

8 Bolding G, Elford J, Sherr L, et al. Survey 2004: behavioural surveillance among gay men using inner London gyms, preliminary report. London: City University, 2004.

9 Pickett J. HAART optimism: is it really the problem with HIV prevention in developed countries? AIDS 2003;18s:S20-S22.

10 Dodds J, Mercey D. Sexual health survey of gay men London 2002. London: Royal Free and University College Medical School, 2003.
11 Dodds J, Mercey D, Parry JV, et al. Increasing risk behaviour and high levels of undiagnosed HIV infection in a community sample of homosexual men. Sex Transm Infect 2004;80:236-40.

12 Little S, Holte S, Routy J, et al. Antiretroviral-drug resistance among patients recently infected with HIV. N Engl J Med 2002;347:385-94.

13 Williamson L, Hart GJ. HIV optimism does not explain increases in high-risk sexual behaviour among gay men in Scotland. AIDS 2004;18:834-6.

14 Elford J. HIV treatment optimism and high risk sexual behaviour among gay men: the population attributable risk. AIDS 2004;(in press).

15 Imrie J. Renewing our commitment. An HIV prevention strategy for Camden and Islington. London: Camden and Islington HIV Prevention Strategy Group, Camden NHS.

16 Elford J, Hart G. If HIV prevention works, why are rates of high risk sexual behaviour increasing among MSM? AIDS Education and Prevention 2003;15:294-308. 